\title{
Discrete-Time Modelling of the Moog Sawtooth Oscillator Waveform
}

\author{
Jussi Pekonen, ${ }^{1}$ Victor Lazzarini, ${ }^{2}$ Joseph Timoney, ${ }^{2}$ Jari Kleimola, ${ }^{1}$ and Vesa Välimäki ${ }^{1}$ \\ ${ }^{1}$ Department of Signal Processing and Acoustics, Aalto University School of Electrical Engineering, P.O. Box 13000, 00076 Aalto, Finland \\ ${ }^{2}$ Digital Sound and Music Technology Group, National University of Ireland, Maynooth, Co. Kildare, Ireland
}

Correspondence should be addressed to Jussi Pekonen, jussi.pekonen@aalto.fi

Received 13 October 2010; Revised 4 January 2011; Accepted 25 February 2011

Academic Editor: Federico Fontana

Copyright (c) 2011 Jussi Pekonen et al. This is an open access article distributed under the Creative Commons Attribution License, which permits unrestricted use, distribution, and reproduction in any medium, provided the original work is properly cited.

\begin{abstract}
Discrete-time modelling strategies of analogue Moog sawtooth oscillator waveforms are presented. Two alternative approaches suitable for real-time implementation are proposed, one modelling the analogue waveform in time domain using phase distortion synthesis and another matching the spectrum of an existing antialiasing sawtooth oscillator to the corresponding analogue spectrum using a first-order IIR post-equalising filter. A parameter estimation procedure for both approaches is explained and performed. Performance evaluation using polynomial fits for the estimated parameters is carried out, and good matches between the model outputs and recorded waveforms are obtained. The best match of the tested algorithms is produced by the phase distortion model and by post-equalising the fourth-order B-spline bandlimited step function sawtooth oscillator.
\end{abstract}

\section{Introduction}

Discrete-time modelling of analogue sound processing units has recently become an active research topic. In addition to the academic interest in the topic, music software companies are continuously creating new plugins and applications that emulate old analogue devices. One of the largest focuses for the research on this topic is the emulation of the subtractive sound synthesis principle of the early electronic synthesisers of 1960s and 1970s. In those synthesisers, a spectrally rich source signal, traditionally one or a sum of several function generator waveforms, such as the sawtooth, the rectangular, and the triangle waveform [1], is filtered with a time-varying and typically resonant lowpass filter.

Due to the modular structure of the analogue synthesisers, the digital emulation of the modules has been split into separate research topics [2]. For the filter module, several models especially for the popular Moog transistor-based voltage-controlled ladder filter [3] have been suggested. These models range from approximative linear circuitbased models $[4,5]$ to models that take into account the nonlinearities characterising the filter sound [6-8] and to models that use a Volterra series representation of the inputoutput relationship of the filter $[9,10]$. Models for another popular synthesiser filter, the diode-based EMS VCS3 filter, have also been suggested [11, 12]. Recently, Huovilainen developed a nonlinear digital model for the second-order resonant lowpass filter that appeared in the Korg MS-20 analogue synthesiser [13].

Whereas the filter models have been based on the behaviour of the analogue circuit, the research on the oscillators has mainly focused on creating bandlimited algorithms that imitate the geometric textbook waveforms (see, e.g., [14-16] for complete list of references). This focus has been justified by the fact that the traditional, trivially sampled, algorithms used to implement the oscillators suffer from harsh aliasing, caused by the discontinuities in the waveform or in the waveform derivative [17].

It has been noted that the output of an analogue oscillator module differs from the respective textbook waveform $[18,19]$. Moreover, they also sound different with the analogue oscillators being less harsh than the textbook waveforms. Figure 1 illustrates this mismatch between the textbook sawtooth waveform and the output of an analogue sawtooth oscillator recorded from a Minimoog Voyager [20] synthesiser's oscillator module (see [21] for the original module circuit design used in its predecessor models) having the fundamental frequency $f_{0}=220.62 \mathrm{~Hz}$. A sampling 
frequency $f_{s}=44.1 \mathrm{kHz}$ was used for the recording. In this case, the textbook sawtooth waveform is a signal containing all harmonics whose amplitudes are inversely proportional to the harmonic index. The recorded waveform does look like the textbook waveform but the rising part of the oscillation period is not linear (see Figure 1(a)). In fact, the rising part in general resembles more a sinusoid than a linear function but not immediately after the waveform reset. The difference can also be seen in the waveform spectrum depicted in Figure 1(b) where the approximately $-6 \mathrm{~dB}$ per octave spectral envelope of the textbook sawtooth is plotted with a dashed line for comparison.

Furthermore, the difference between the textbook waveform and the analogue oscillator output depends on the fundamental frequency. This can be seen in Figure 2 where the waveform and the spectrum of the Minimoog Voyager sawtooth output are plotted for $f_{0}=2.096 \mathrm{kHz}$. There are small differences in the waveforms (compare Figures 1(a) and 2(a)), but the differences can be seen more clearly in the spectrum plot. The higher harmonics of the high $f_{0}$ sawtooth (crosses in Figure 2(b)) are lower in magnitude than the respective harmonics of the low $f_{0}$ waveform (circles in Figure 2(b), scaled in frequency). Moreover, the spectral envelope differs from the low $f_{0}$ envelope (dash-dotted line in Figure 2(b), shifted in magnitude so that the $0 \mathrm{~dB}$ level is at the fundamental frequency $2.096 \mathrm{kHz}$ ).

So far, only two papers have dealt with the topic of discrete-time modelling of an analogue audio oscillator module. De Sanctis and Sarti derived a wave-digital filter model for an astable multivibrator circuit in [22]. The astable multivibrator discussed in [22] is based on operational amplifiers whereas analogue synthesisers utilise discrete components in their oscillator circuits more often. The only model for the output waveform of such a circuit was proposed in [19]. This introduced an ad-hoc Moog oscillator model consisting of a scaled and shifted quarter of a sine wave starting from -1 and was reset once the waveform reached +1 . In other words, the model uses a part of a sine wave whose frequency one fourth of the target $f_{0}$. However, the rapid transition of this simplified model results in large aliasing as in the case of trivial sampling. A modification to the model that utilises a second-order polynomial correction function approximating a bandlimited step function [14] at the waveform reset was also suggested in [19]. With this modification the aliasing was greatly reduced. It should be noted that the model proposed in [19] was actually an example of the waveslicing technique discussed in that paper and that the example happens to produce a waveform similar to the Moog sawtooth oscillator.

In this paper, alternative $f_{0}$-dependent approaches to simulate the Moog sawtooth oscillator are proposed. A set of recorded sawtooth waveforms from the Minimoog Voyager [20] is used as a reference. The reference signals were recorded directly from the oscillator module output without feeding them through the filter module using an M-Audio Quattro audio interface with sampling frequency $44100 \mathrm{KHz}$ and resolution of 16 bits. Two novel discrete-time modelling strategies well suited for real-time implementation are proposed. The proposed strategies are based on signal modelling that tries to mimic the recorded waveform by synthesising a signal that has similar signal characteristics. This signalbased approach enables the use of readily available tools in the synthesis, and it avoids design-dependent issues, for example, the discretisation method and delay-free loops, present in circuit-based modelling.

The remainder of this paper is organised as follows. First, Section 2 introduces direct waveform modelling using phase distortion synthesis. Then, a more general postprocessing equalising filter approach for existing antialiasing oscillator algorithms is presented in Section 3. The methods are evaluated by determining their spectral error from the reference signals. Finally, Section 4 concludes the paper.

\section{Waveform-Based Modelling Using Phase Distortion Synthesis}

The first discrete-time model of the Moog sawtooth waveform oscillator proposed here is based on direct time-domain modelling of the target waveform using phase distortion (PD) synthesis [23-26]. In PD synthesis, the normally linear $f_{0}$-dependent phase trajectory $\phi_{\text {lin }}(t)$ of a sinusoid is modified with a nonlinear shaping function $f(x)$, that is, the phase distorted sinusoid is given by

$$
y_{\mathrm{PD}}(t)=\sin \left(f\left(\phi_{\text {lin }}(t)\right)+\phi_{0}\right),
$$

where $t$ is time and $\phi_{0}$ is the initial phase. This approach is effectively the same as phase (or frequency) modulation as the phase shaping function can be decomposed into a linear part and a time-varying component [24-26] as

$$
f\left(\phi_{\text {lin }}(t)\right)=\phi_{\text {lin }}(t)+\phi_{\text {mod }}(t)=2 \pi t+\phi_{\bmod }(t),
$$

where $\phi_{\text {mod }}(t)$ is the time-varying component. For the PD sawtooth waveform originally described in [23], $\phi_{0}=-\pi / 2$ and $\phi_{\bmod }(t)$ is given as a skewed sawtooth (triangular) function expressed as

$$
\phi_{\text {mod,saw }}(t)=(\pi-2 \pi P) \times \begin{cases}\frac{t}{P} & (t \bmod 1)<P, \\ \frac{1-t}{1-P} & (t \bmod 1) \geq P,\end{cases}
$$

where $P \in[0,1]$ is the fraction of the period during which the sawtooth function is rising $[24,25]$.

Applying (2) and (3) to (1) yields

$$
\begin{aligned}
y_{\mathrm{PD}, \mathrm{saw}}(t) & =\sin \left(2 \pi t+\phi_{\text {mod,saw }}(t)-\frac{\pi}{2}\right) \\
& =-\cos \left(2 \pi t+\phi_{\text {mod,saw }}(t)\right),
\end{aligned}
$$

which produces a waveform that resembles the sawtooth waveform. With $P<.5$, the maximum of the waveform is closer to the beginning of oscillation period, and when $P>.5$, the maximum is closer to the end of the period. With $P=.5$, the modulation function $\phi_{\text {mod,saw }}(t)$ does not have any effect since it will be zero at all time instants. 


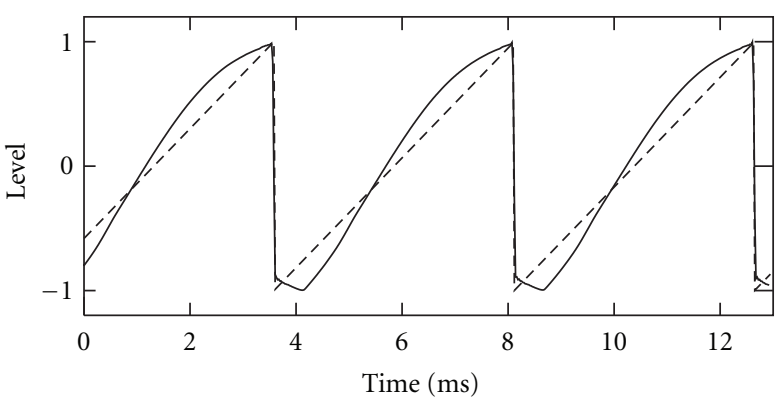

(a)

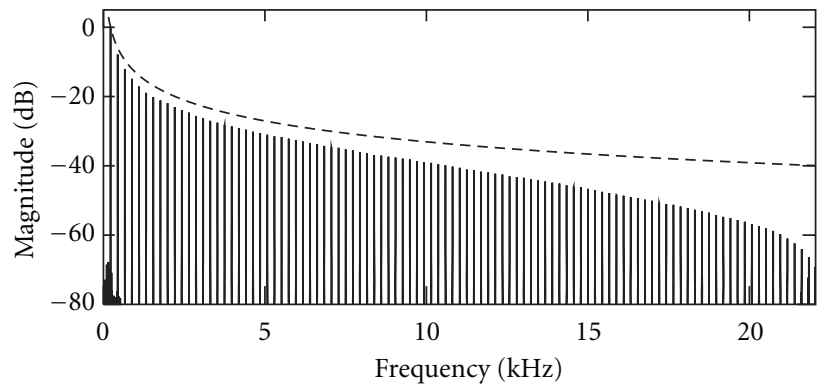

(b)

Figure 1: (a) Recorded Moog sawtooth waveform having the fundamental frequency $f_{0}=220.62 \mathrm{~Hz}$. The spectrum of the waveform is shown in (b) together with the approximately $-6 \mathrm{~dB}$ per octave spectral envelope of the textbook sawtooth waveform (dashed line). Sampling frequency $f_{\mathrm{s}}=44.1 \mathrm{kHz}$ was used for the recording. In (a), the dashed line represents the textbook sawtooth waveform.

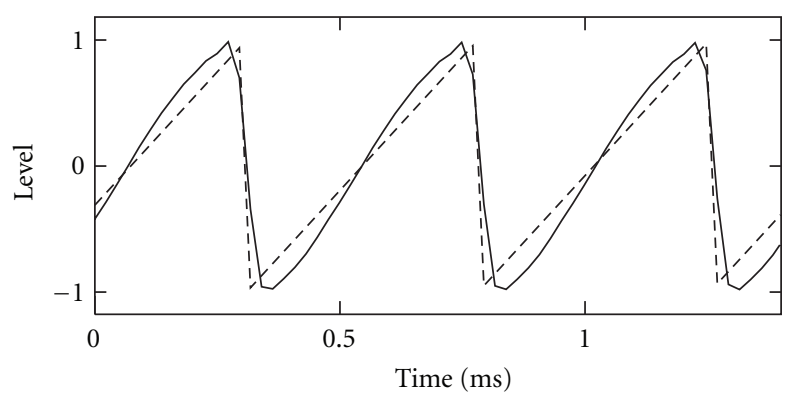

(a)

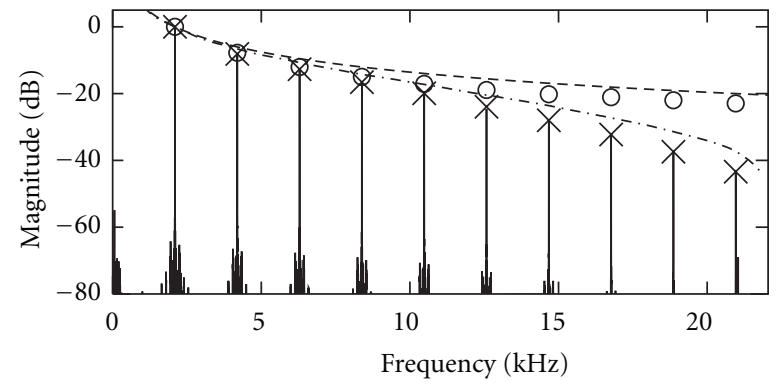

(b)

Figure 2: (a) Waveform and (b) spectrum of the recorded Moog sawtooth having $f_{0}=2.096 \mathrm{kHz}$. In (b), the crosses indicate the magnitudes of the waveform harmonics, the circles represent the magnitudes of the frequency-scaled harmonics of the recorded sawtooth waveform having $f_{0}=220.62 \mathrm{~Hz}$, and the dash-dotted line is the magnitude-shifted spectral envelope of the sawtooth oscillator output for $f_{0}=$ $220.62 \mathrm{~Hz}$. The dashed line represents the waveform and the spectral envelope of the textbook sawtooth in (a) and (b), respectively.

The model proposed in [19] can be understood as a special case of the PD synthesis model described above. That model has the PD model parameters $\phi_{0}=0$ and $\phi_{\text {mod }}(t)=-7 \pi t / 4+\Delta(t)$, where $\Delta(t)$ is an impulse-trainlike function that modifies the phase of the two samples around the waveform reset. The difference between the model of [19] and the general PD model discussed in this paper is demonstrated in Figure 3, where the phase-shaping functions of the two models are plotted.

2.1. Model Parameter Estimation. In order to produce PD sawtooth waveforms that resemble the Moog sawtooth waveform, the model parameter $P$ must be fitted to produce replicas of the target waveforms that are as close as possible. The model parameter can be estimated from the phase trajectory of the reset portion of a recorded waveform. Since the recorded waveforms have their maxima close to the end of the oscillation period (see Figures 1(a) and 2(a)), the phase trajectory of the reset portion can be approximated to be linear as given by

$$
\begin{aligned}
\hat{\phi}_{\text {reset }}(t) & =2 \pi t+(\pi-2 \pi P) \frac{1-t}{1-P} \\
& =2 \pi\left(\left(1-\frac{1-2 P}{2-2 P}\right) t+\frac{1-2 P}{2-2 P}\right) .
\end{aligned}
$$

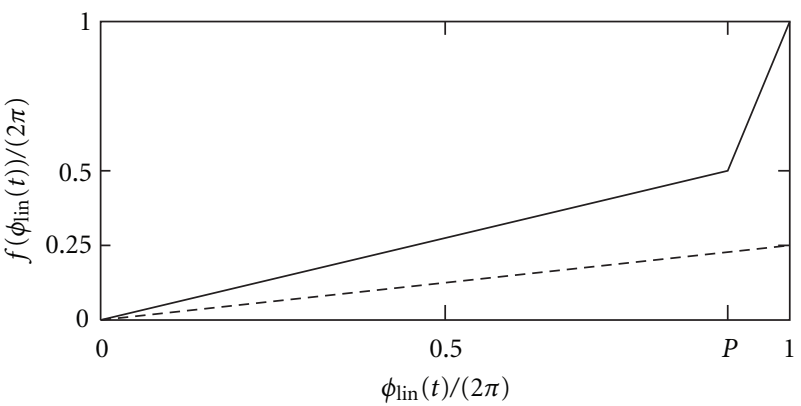

Figure 3: The phase-shaping functions of the ad-hoc model without the waveform reset modification presented in [19] (dashed line) and the phase distortion model discussed in this paper (solid line).

Now, the model parameter $P$ can be estimated by fitting a linear approximation to the phase trajectory of the reset part of the recorded waveforms. By choosing at least two samples from the reset part and by applying the inverse cosine function to the negated values of these selected points (with a caution on phase wrapping performed by the inverse cosine function), a set of phase data points are obtained. For 


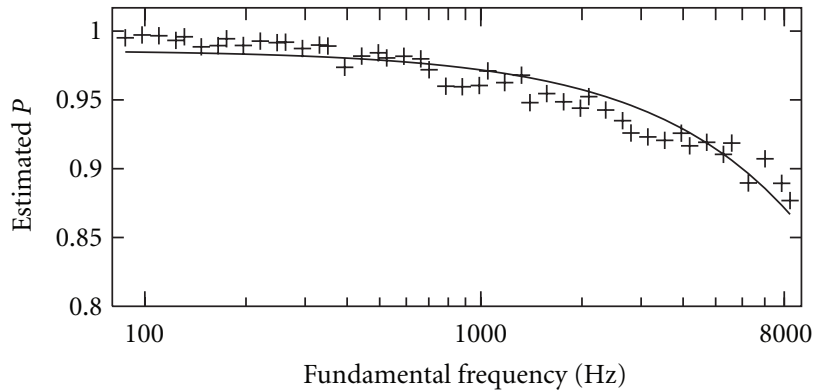

(a)

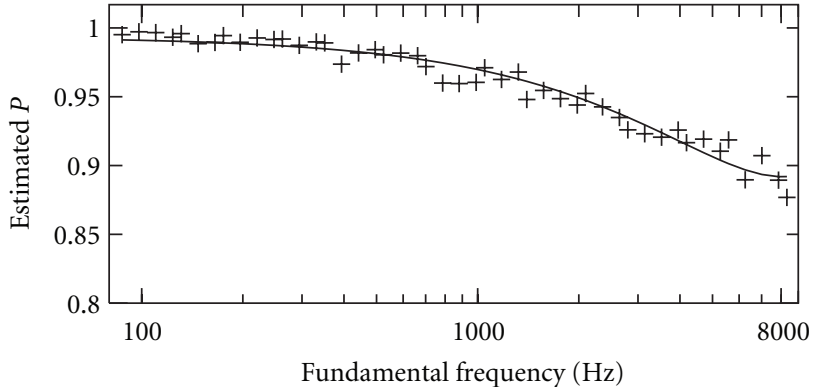

(b)

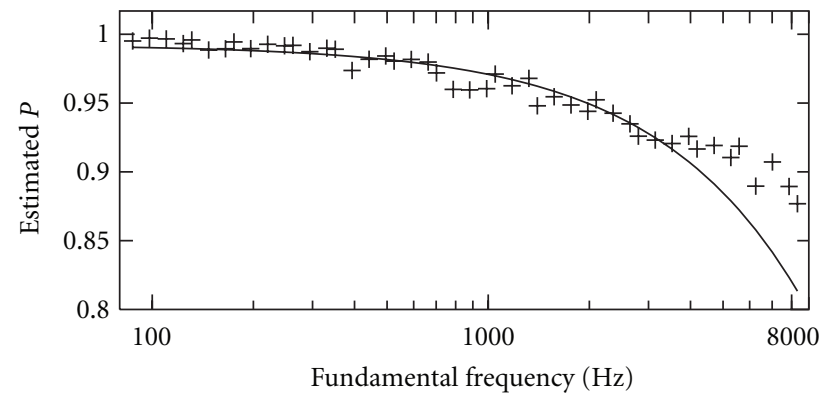

(c)

Figure 4: Phase distortion (PD) model parameter estimate means (plus signs) and (a) a first-order and (b) a second-order fit to the estimate data using all data points. (c) A first-order fit to the estimate data using data points below $4 \mathrm{kHz}$.

these phase data points, a linear fit can be computed, and from the coefficients of the fit an estimate for $P$ is obtained using the relations given in (5). In order to improve the estimate, this procedure can be carried out for several reset parts, and the mean of these estimates can be used as the model parameter for that fundamental frequency.

Using the approach described above, the model parameter $P$ was estimated from five reset parts of 47 recorded Moog sawtooth waveforms having different $f_{0}$ in the range from $86 \mathrm{~Hz}$ to $8.3 \mathrm{kHz}$. The estimated model parameter data as a function of the fundamental frequency is shown in Figure 4 with plus signs. The data shows a clear dependency on $f_{0}$, being close to one at low frequencies and the estimated $P$ decreases as the fundamental frequency increases. In order to analyse the dependency, low-order polynomial fits for the estimated data in the least-squares sense were sought. In Figure 4(a), a first-order fit of the estimated data is plotted using the whole data set, and a second-order fit is plotted in Figure 4(b). Both low-order polynomials generally provide a good match to the estimated $P$, but at low fundamental frequencies, which are more common in musical signals, the first-order fit differs from the estimated data more than the second-order fit. A first-order fit was also made only for the estimated data points below $4 \mathrm{kHz}$. This linear fit, given by

$$
\widehat{P}\left(f_{0}\right)=0.9924-0.00002151 f_{0},
$$

is plotted in Figure 4(c), showing an excellent match at low frequencies while producing smaller $P$ values than the estimated data at high frequencies.
When the polynomial approximation is used, the $f_{0}$ dependent model parameter $P$ can be computed directly from the fundamental frequency with a lower computational cost and memory consumption than in the case where the estimated parameter values are tabulated. In the table-based parameter computation, the real-time control of the model parameter is not as trivial as with the polynomial approximation when the fundamental frequency is modulated with a low frequency oscillator or an envelope. In that case, the time-varying fundamental frequency needs to be mapped to a table index using a nonlinear function which is computationally more costly than the evaluation of a low-order polynomial that utilises the fundamental frequency value perse. Furthermore, the table-based approach consumes more memory than a polynomial approximation that has only a few constant coefficients which do not need to be tabulated at all. On the other hand, the deviation of the linear approximation of (6) from the estimated parameter values at high fundamental frequencies is in fact advantageous due to lowered aliasing. The farther $P$ is from .5 , the faster the waveform reset is and the more aliasing the PD sawtooth will contain.

2.2. Model Evaluation. Figure 5 shows the match of the PD model output to the recorded Moog sawtooth oscillator output with fundamental frequency $f_{0}=220.62 \mathrm{~Hz}$ using the estimated value of model parameter $P$. In Figures 5(a) and 5(b), the waveform and the spectrum of the PD model are drawn, respectively, together with the waveform and the spectral envelope of the recorded signal (dashed line). The 


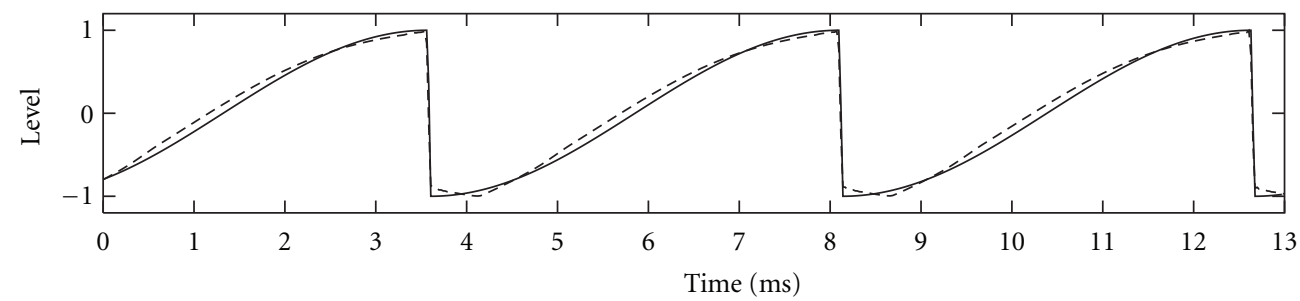

(a)

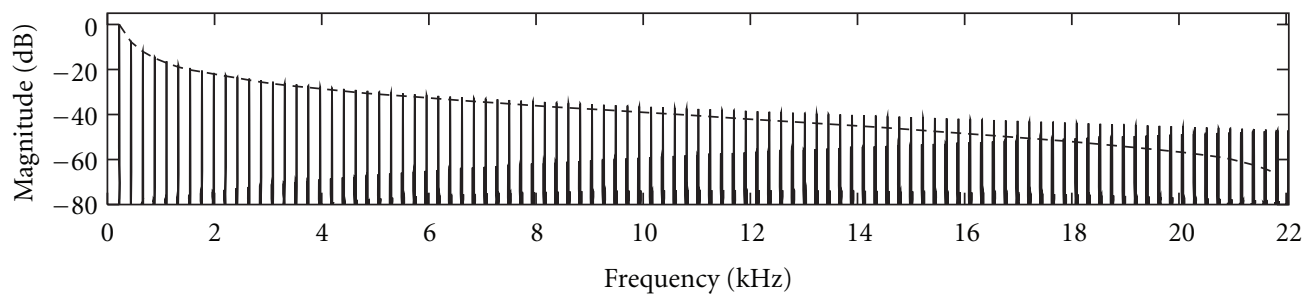

(b)

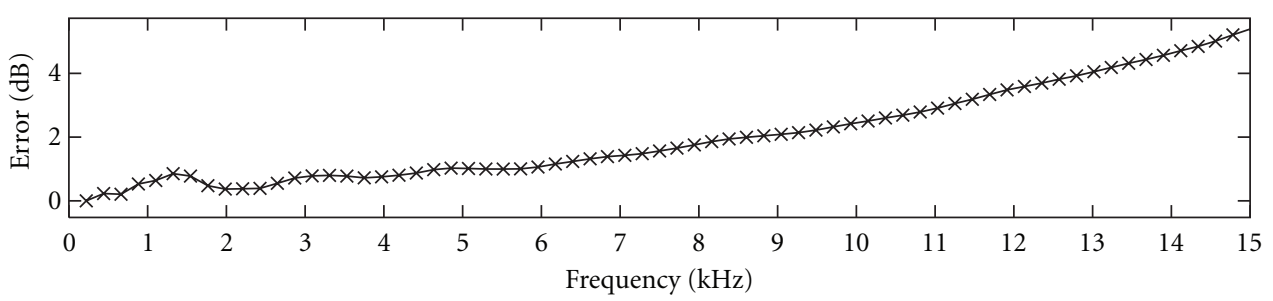

(c)

Figure 5: (a) Waveform, (b) the spectrum, and (c) the harmonic magnitude error of the PD model of the Moog sawtooth oscillator output, that is, the difference between the model output harmonic magnitudes and the magnitudes of the recorded signal harmonics, at $f_{0}=$ $220.62 \mathrm{~Hz}$. As the model parameter, the estimated value was used. The waveform and the spectral envelope of the recorded signal is drawn with a dashed line in (a) and (b), respectively, for comparison.

PD model provides a faithful imitation of the recorded signal, differing mainly at very high frequencies. Some aliasing can be seen in Figure 5(b), but it is mainly at high frequencies where human hearing is less sensitive [27]. Figure 5(c) shows the harmonic magnitude error, that is, the difference between the model output harmonic magnitudes and the magnitude of the recorded harmonics, for harmonic frequencies below $15 \mathrm{kHz}$. The harmonic magnitude error above $15 \mathrm{kHz}$ is not of great interest because human hearing is very insensitive above this frequency [27]. As one can see, the harmonic magnitude error below $15 \mathrm{kHz}$ is within a few decibels at all harmonics.

Figure 6 shows the match of the PD synthesis model to the recorded Moog sawtooth oscillator with $f_{0}=2.096 \mathrm{kHz}$ using the estimated model parameter. The waveform and the spectrum in Figures 6(a) and 6(b) show a larger mismatch to the recorded waveform than in the case of low $f_{0}$ (see Figures 5(a) and 5(b)). The harmonic magnitude error, shown in Figure 6(c), is slightly larger than in the low $f_{0}$ example (see Figure 5(c)). Moreover, the output of the high $f_{0}$ PD model contains more aliasing than the low $f_{0}$ model, as one could expect. However, the aliasing in this example case is focused close to the harmonics, and it is inaudible due to the frequency masking phenomenon [27]. It should be noted that this focusing of aliasing close to the harmonic components is not characteristic to all fundamental frequencies, and hence conclusions on the audibility of aliasing at arbitrary fundamental frequencies should not be drawn from this example.

From Figures 5 and 6, one can conclude that the harmonic magnitude error depends on the fundamental frequency. In order to evaluate this, the error of the harmonic components below $15 \mathrm{kHz}$ was computed for the fundamental frequencies used in the PD model parameter estimation and the evaluation results are shown in Figure 7. In Figure 7(a), the root mean squared error (RMSE) of the PD model is plotted for all tested fundamental frequencies with a solid line for the linear approximation of (6). In addition, the RMSE of the PD model using the estimated model parameter values is plotted with crosses for comparison in Figure 7(a). It can be noted that the polynomial approximation of the model parameter produces an error comparable to the error obtained with the estimated values, and hence the use of the polynomial approximation of $P$ provides accuracy-wise as a good match as the tabulated estimates to the recorded waveform. The RMSE of the PD model is small (around $-50 \mathrm{~dB})$ at low fundamental frequencies and becomes larger when $f_{0}$ is increased.

However, since the RMSE is a measure of the averaged error at the harmonic components, it may ignore possibly 


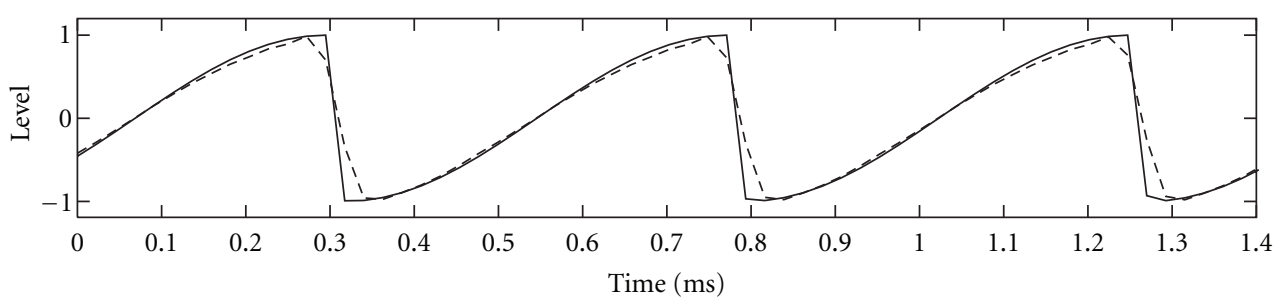

(a)

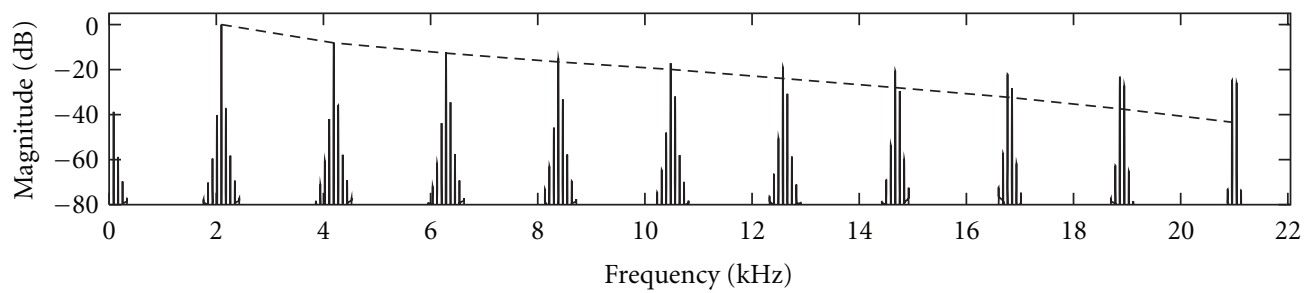

(b)

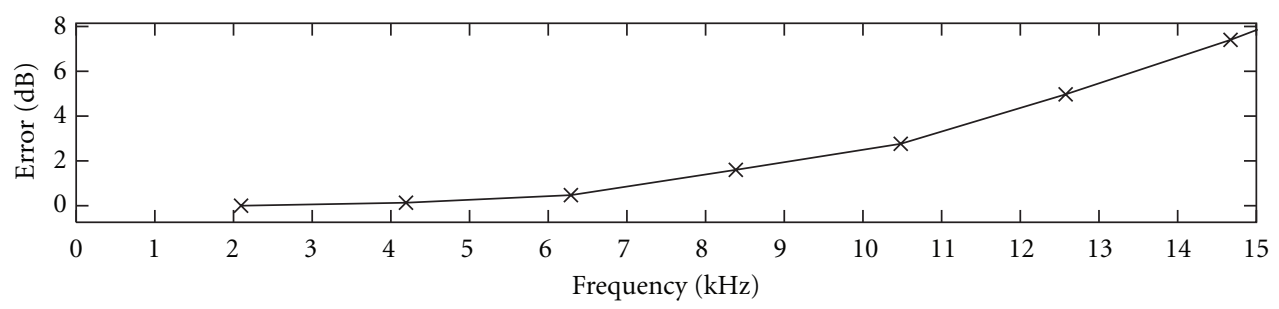

(c)

FIGURE 6: (a) Waveform, (b) the spectrum, and (c) the harmonic magnitude error of the PD model of the Moog sawtooth oscillator output at $f_{0}=2.096 \mathrm{kHz}$. The waveform and the spectral envelope of the recorded signal are again drawn with dashed line in (a) and (b), respectively.

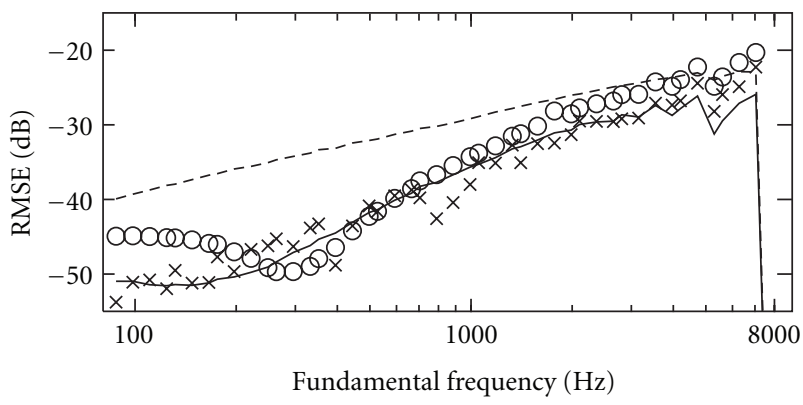

(a)

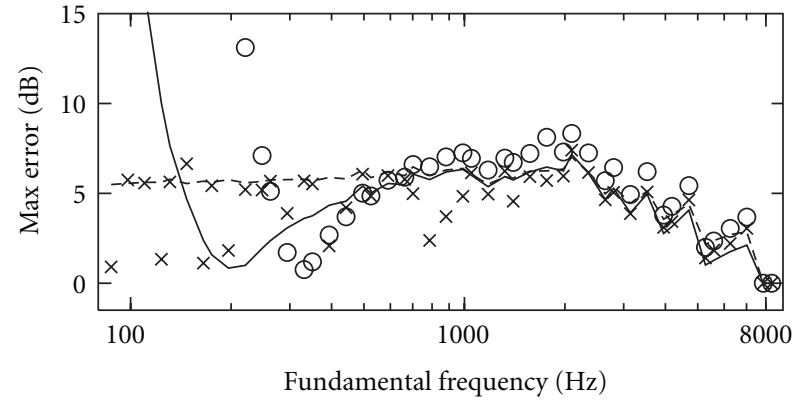

(b)

Figure 7: (a) Root mean squared error (RMSE) of the harmonics and (b) the maximum absolute harmonic magnitude error of the PD model (solid line) and the reset-corrected model of [19] (dashed line) as a function of $f_{0}$ between $86 \mathrm{~Hz}$ and $8.3 \mathrm{kHz}$. The RMSE and the maximum absolute harmonic magnitude error of PD models that use the estimated parameter values and a single parameter value estimated for $f_{0}=524 \mathrm{~Hz}$ at all frequencies are plotted with crosses and circles, respectively, for comparison.

large errors of individual harmonics. In order to evaluate this aspect too, the maximum absolute harmonic magnitude error was also computed for the harmonics below $15 \mathrm{kHz}$. Figure 7(b) shows the maximum harmonic error of the PD model as a function of the fundamental frequency with a solid line for the polynomial approximation of the model parameter and with crosses for the estimated $P$ values.

Now, the PD oscillator that uses the polynomial approximation of the model parameter produces a very large error at low fundamental frequencies, whereas the estimated parameter values yield a smaller maximum absolute harmonic magnitude error. However, above $150 \mathrm{~Hz}$, the polynomial approximation yields an error that is comparable to the estimated values, being within a few decibels. At low fundamental frequencies, the large error of the polynomial approximation oscillator is produced by one of the highest harmonics in the tested bandwidth. This happens because $P$ is very close to one at low frequencies (see (6)) which 
effectively results in a faster waveform reset and increased high-frequency content. Since the spectral envelope of the Moog sawtooth clearly differs from the spectral envelope of the textbook sawtooth (see Figures 1 and 2), the model produces larger harmonic magnitude error at one of the highest harmonics. However, since the waveform has many harmonics at low fundamental frequencies, the averaging process of the RMSE measure decreases the significance of these large individual errors.

On the other hand, it would be advantageous to use a single model parameter at all fundamental frequencies as it would alleviate the computation of the PD model parameter value from the synthesis control data (namely $f_{0}$ in this case). However, as indicated with circles in Figure 7, the PD sawtooth that uses a single parameter estimated for $f_{0}=$ $524 \mathrm{~Hz}$ at all frequencies has larger RMSE and maximum absolute harmonic magnitude error than the polynomialbased oscillator, except in the frequency range from $250 \mathrm{~Hz}$ to $400 \mathrm{~Hz}$. Therefore, the model parameter needs to be $f_{0^{-}}$ dependent, and the best match to the reference waveforms with the lowest possible computational cost is obtained by using the linear approximation of (6) of the PD model parameter $P$.

Figure 7 also shows the corresponding RMSE and maximum absolute harmonic magnitude error for the resetcorrected model of [19] with dashed lines. As one can observe, the RMSE of this model is worse than that of the proposed PD model. However, the maximum harmonic error is approximately comparable to the error of the PD model proposed here. At very low frequencies, where the maximum harmonic error of the PD model is large, the resetcorrected model of [19] has lower error, but above $150 \mathrm{~Hz}$ the error is at least as large as that of the PD model.

From Figures 5, 6, and 7 it can be concluded that the PD model that uses the polynomial approximation of the model parameter can be used to produce waveforms that match well to the recorded Moog sawtooth oscillator waveforms. However, the accuracy of the model depends on the fundamental frequency as with some frequencies the model produces an excellent match, while with other frequencies the match is slightly poorer. Nevertheless, the PD model with the polynomial approximation of the $f_{0}$ dependent parameter $P$ produces a good match to the target using a simplified and efficient control.

\section{Post-Equalisation of Antialiasing Oscillator Outputs}

Whereas the phase distortion model discussed in Section 2 was based on time-domain modelling of the Moog sawtooth oscillator waveform, the second model proposed here is based on frequency-domain matching. This approach uses an existing antialiasing sawtooth oscillator algorithm, which models the ideally bandlimited sawtooth and filters the output of that algorithm with a low-order filter to produce a spectrum that is a close replica of the spectrum of the recorded signal. In other words, this model is a sourcefilter approach whose filter modifies the spectrum of the antialiasing oscillator towards the spectrum of the analogue oscillator module.

3.1. Antialiasing Oscillator Used as the Source. Here, five different antialiasing oscillator algorithms are considered. These oscillator algorithms are chosen to represent a variety of currently available approaches, and they are briefly reviewed next.

3.1.1. Ideally Bandlimited Sawtooth Oscillator. As the first approach, an ideally bandlimited oscillator algorithm is considered. This approach produces only the harmonics below the Nyquist limit, the number of which is given by $K=\left\lfloor f_{s} /\left(2 f_{0}\right)\right\rfloor$ with $\lfloor x\rfloor$ denoting the floor function, that is, rounding to the integer part. The ideally bandlimited sawtooth waveform is then given as

$$
y_{\mathrm{bl}}(n)=-\frac{2}{\pi} \sum_{k=1}^{K} \frac{\sin \left(2 \pi k f_{0} n / f_{s}\right)}{k},
$$

where $n$ is the sample index, which is obtained by taking the $K$ first terms from the Fourier series representation of the continuous-time sawtooth waveform. There are a few alternative approaches to synthesise the ideally bandlimited sawtooth, the most familiar ones being additive [28] and wavetable [29] synthesis.

3.1.2. Third-Order B-Spline Polynomial BLIT Sawtooth Oscillator. The second algorithm considered here is the thirdorder B-spline polynomial bandlimited impulse train (BLIT) algorithm [15]. The idea in the BLIT algorithm is to generate a sequence of bandlimited impulses that is then integrated to produce the bandlimited waveform [30]. Since the ideal bandlimited impulse is the well-known sinc function which is infinitely long [30], it is approximated in practical realisations, resulting in an approximately bandlimited impulse which has aliasing mainly at high frequencies. The thirdorder B-spline BLIT algorithm uses the third-order B-spline basis function as the approximation of the bandlimited impulse [15]. The basis function is synthesised with a thirdorder FIR filter given by

$$
H_{b_{3}}(z, d)=\sum_{k=0}^{3} b_{3}(k, d) z^{-k},
$$

where $d$ is the fractional delay from the discontinuity to the sample following it and $b_{3}(k, d), k=0,1,2,3$ are the filter coefficients as a function of $d$ as given in Table 1. Note that these coefficients differ from those given in [15] due to the fact that here the impulse needs to be multiplied by -2 in order to get the desired waveform reset. This filter is triggered at each discontinuity according to the delay the impulse needs to be shifted in time. By integrating the resulting impulse train, an approximation of the bandlimited sawtooth waveform is obtained. In this paper, a second-order leaky integrator, expressed as [31]

$$
H_{\text {int }}(z)=\frac{\pi(1-0.9992)\left(1-z^{-1}\right)}{2\left(1-0.9992 z^{-1}\right)\left(1-0.9992 z^{-1}\right)},
$$


TABLE 1: Filter coefficients for the third-order B-spline bandlimited impulse train (BLIT) synthesiser as a function of the fractional delay d.

\begin{tabular}{lc}
\hline$k$ & $b_{3}(k, d)$ \\
\hline 0 & $-d^{3} / 3$ \\
1 & $d^{3}-d^{2}-d-1 / 3$ \\
2 & $-d^{3}+2 d^{2}-4 / 3$ \\
3 & $d^{3} / 3-d^{2}+d-1 / 3$ \\
\hline
\end{tabular}

is used. This integrator suppresses the DC component which would otherwise have to be added to every sample of the impulse train.

3.1.3. Fourth-Order B-Spline Polynomial BLEP Sawtooth Oscillator. As the third algorithm, an extension of the thirdorder B-spline BLIT algorithm, the fourth-order B-spline polynomial bandlimited step function (BLEP) algorithm [32], is used. In the BLEP algorithm, the integration required by the BLIT algorithm (see above) is performed beforehand [31]. Integrating the bandlimited impulse yields an approximation of a bandlimited step function, a bandlimited representation of a waveform discontinuity [31]. As the ideal bandlimited impulse, the sinc function, is infinitely long, so is the ideal bandlimited step function [32]. Therefore, for practical realisations an approximation of the ideal bandlimited step function needs to be computed. In the fourth-order B-spline BLEP algorithm, the third-order Bspline basis function is analytically integrated with respect to time [32] and a fourth-order polynomial approximation of the ideal step function is obtained. Typically the resulting function is not applied as is, instead the difference between the bandlimited step function and the nonbandlimited step function is added onto the trivial nonbandlimited waveform at each discontinuity $[14,33]$. That is, the impulse response of a third-order FIR filter given by

$$
H_{B_{4}}(z, d)=\sum_{k=0}^{3} B_{4}(k, d) z^{-k},
$$

where $B_{4}(k, d), k=0,1,2,3$ are the fourth-order B-spline polynomial BLEP filter coefficients as a function of the fractional delay as given in Table 2, is summed onto the output of the trivial sawtooth oscillator at each discontinuity as a correction function [32]. Again, the coefficients of the filter given in [32] are scaled by -2 .

\subsubsection{Second- and Fourth-Order DPW Sawtooth Oscillators.} The remaining two approaches are two cases of the differentiated polynomial waveform (DPW) algorithm [16]. In DPW, the basic idea is to reduce the aliasing of a sawtooth waveform by modifying the spectral tilt of the signal to be sampled [ 16 , 34]. In practice this is implemented by integrating the linear ramp function of the sawtooth waveform. Each integration reduces the spectral envelope by approximately $-6 \mathrm{~dB}$ per octave, for example, the first integral of the linear ramp, that is, a parabola, has an approximately $-12 \mathrm{~dB}$ per octave spectral envelope compared to the approximately $-6 \mathrm{~dB}$
TABLE 2: Filter coefficients for the fourth-order B-spline bandlimited step function (BLEP) algorithm as a function of the fractional delay $d$.

\begin{tabular}{lc}
\hline$k$ & $B_{4}(k, d)$ \\
\hline 0 & $-d^{4} / 12$ \\
1 & $d^{4} / 4-d^{3} / 3-d^{2} / 2-d / 3-1 / 12$ \\
2 & $-d^{4} / 4+2 d^{3} / 3-4 d / 3+1$ \\
3 & $d^{4} / 12-d^{3} / 3+d^{2} / 2-d / 3+1 / 12$ \\
\hline
\end{tabular}

per octave envelope of the linear ramp [16]. Sampling this tilted waveform suppresses the aliasing. Now, the sampled polynomial waveform, which can be computed in advance, needs to be differentiated to retain the original spectral envelope of the sawtooth waveform $[16,34]$. The number of required differentiators is equal to the number of integration steps performed, that is, if the polynomial waveform to be sampled is the $N$ th integral of the linear ramp, the resulting signal needs to be differentiated $N$ times in order to obtain the sawtooth waveform [16].

Here, we consider the second-order DPW algorithm which samples the square of the trivial sawtooth waveform $s(t)$,

$$
x_{\mathrm{DPW}, 2}(t)=s(t)^{2},
$$

and filters it with the first-order differentiator [34],

$$
H_{\text {diff }}(z)=1-z^{-1},
$$

and the fourth-order DPW algorithm which samples a fourth-order polynomial

$$
x_{\mathrm{DPW}, 4}(t)=s(t)^{4}-2 s(t)^{2}
$$

and filters it with a cascade of three first-order differentiators [16]. In addition, both approaches need a post-scaling operation that retains the waveform amplitude suppressed by the nonideal differentiation $[16,34]$.

3.1.5. Note on the Ideally Bandlimited Sawtooth Oscillator. It should be noted that the ideally bandlimited approach could be used to synthesise the analogue sawtooth directly using the levels and phases of the analogue waveform harmonics. With the other approaches discussed in this paper this is not possible directly since they have spectra that are characteristic to the algorithm and not controllable. Here, the ideally bandlimited oscillator that has exactly the levels of the textbook sawtooth is used as a reference example of an oscillator that synthesises the bandlimited textbook sawtooth perfectly. In this paper, the ideally bandlimited oscillator is implemented using additive synthesis. However, note that since the computational cost of additive synthesis oscillator is considered large (inversely proportional to the fundamental frequency), it limits both the polyphony and the computing power available for other tasks such as filtering and effects processing. Therefore, it is not suitable for real-time implementation in general. 


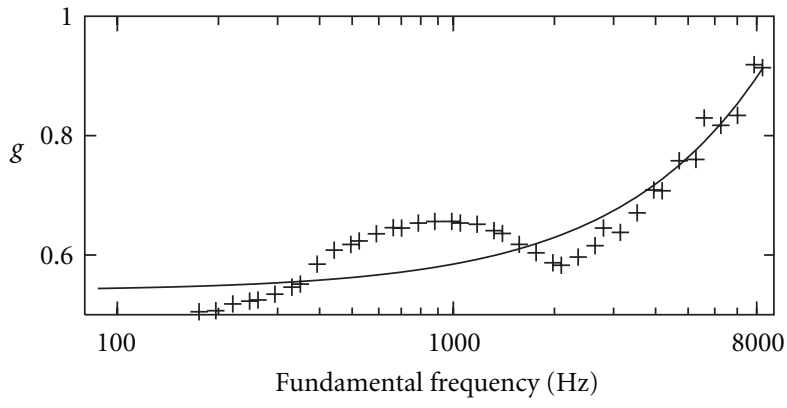

(a)

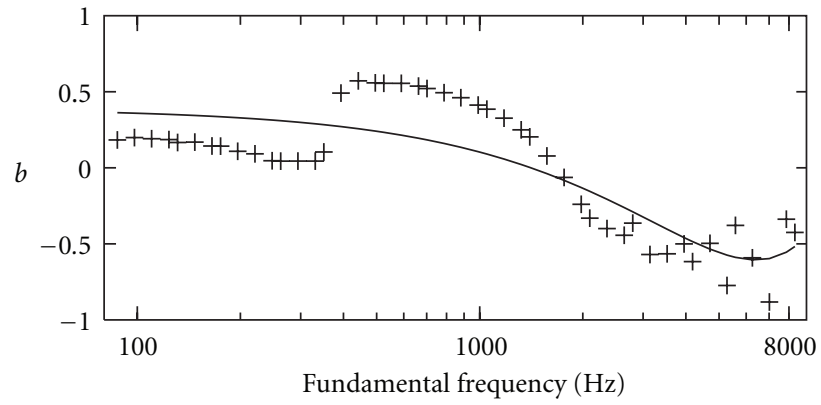

(b)

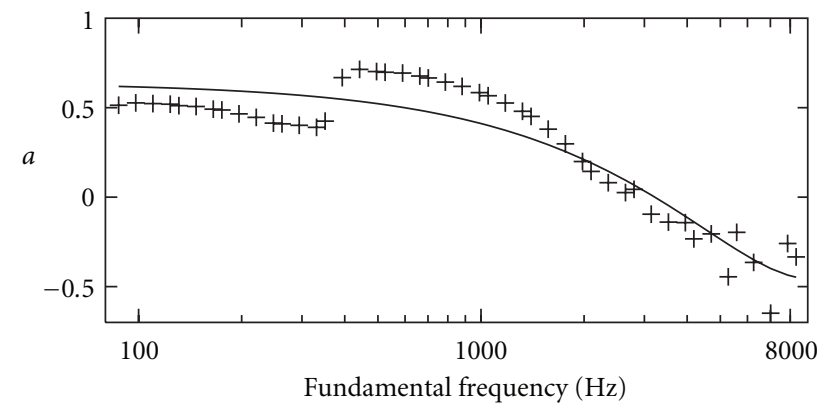

(c)

FIGURE 8: First-order filter parameter estimates (plus signs) for the ideally bandlimited oscillator: (a) the gain factor $g$, (b) the filter zero $b$, and (c) the filter pole $a$. The solid lines represent the polynomial approximations of the filter parameters.

3.2. Filter Estimation. The oscillator algorithms described above obviously have a spectral envelope that differs from the spectral envelope of the Moog sawtooth oscillator. In order to modify the envelope of an oscillator to match the target envelope, the oscillator output is filtered with a loworder filter. For the filter estimation, a frequency-weighted least-squares minimisation of the magnitude response error at the waveform harmonic frequencies was performed [35]. As the frequency-weighting function, a piecewise constant function which has unity weight for all the other harmonic components except the nine lowest harmonics, which had a weight of 10, and the fundamental frequency, which had a weight of 100 , was used. With this weighting function, the first ten components, which contribute quite a lot to the timbral perception, are emphasised.

A first-order IIR filter given by

$$
H_{\mathrm{eq}}(z)=g \frac{1-b z^{-1}}{1-a z^{-1}}
$$

was considered as the post-equalising filter due to its simplicity. The parameters $g, b$, and $a$, the gain factor, the filter zero, and the filter pole, respectively, of $H_{\mathrm{eq}}(z)$ were estimated from the recorded data using the approach described above.

The estimated filter parameters for the ideally bandlimited oscillator are shown in Figure 8 with plus signs. Again, the filter parameter exhibits dependency on the fundamental frequency. The gain factor $g$ (see Figure 8(a)) shows an approximately linear dependency on $f_{0}$, and the filter zero $b$ and pole $a$ show approximately quadratic dependency (see Figures $8(\mathrm{~b})$ and $8(\mathrm{c})$, resp.). For the estimated data, a firstorder fit for the gain factor and second-order fits for the filter zero and pole were computed in the least-squares sense. These approximations are also shown in Figure 8 with solid lines. As one can observe, they provide a good match to the estimated parameter data. These parameter fits, expressed as

$$
\{\hat{g}, \hat{b}, \hat{a}\}\left(f_{0}\right)=c_{2} f_{0}^{2}+c_{1} f_{0}+c_{0}
$$

where $c_{i}$ is the $i$ th fit coefficient, can be used to control the filter parameters as a function of $f_{0}$. The fitted coefficient values for the ideally bandlimited oscillator are given in Table 3 .

For the other oscillator approaches, similar relationships between the fundamental frequency and the filter parameters were found. This is indicated in Figure 9 for the thirdorder B-spline BLIT and the fourth-order B-spline BLEP sawtooth oscillators and in Figure 10 for the second-order DPW and the fourth-order DPW sawtooth oscillators. The respective polynomial filter parameter fit coefficients are given in Table 4. Again, the polynomial fits match quite well to the estimated parameter data. The fits are better with the third-order B-spline BLIT, the fourth-order B-spline BLEP, and the fourth-order DPW algorithm than with the ideally bandlimited and the second-order DPW algorithm. Moreover, as with the ideally bandlimited algorithm, the polynomial fits are better for the filter zero and pole than for the filter gain. However, these small mismatches of the polynomial fits to the estimated data do not produce very severe errors in the output as discussed next. 


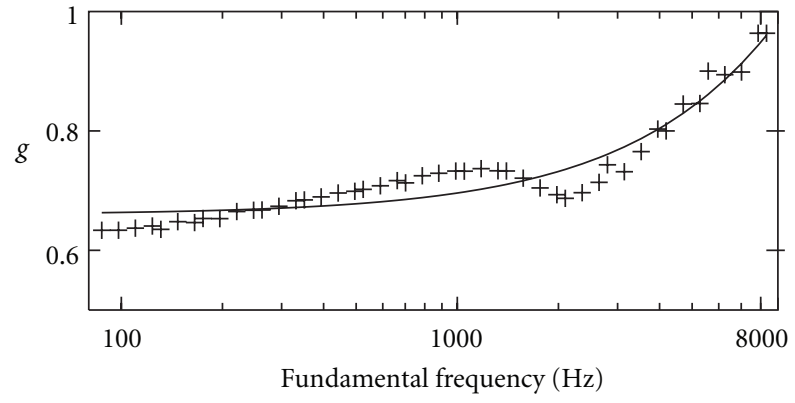

(a)

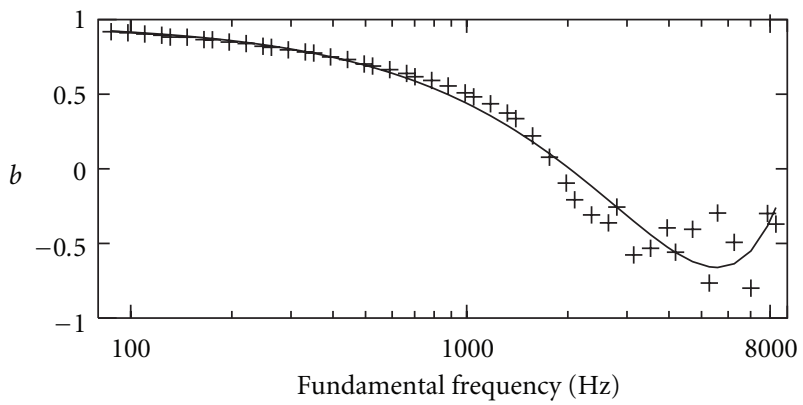

(c)

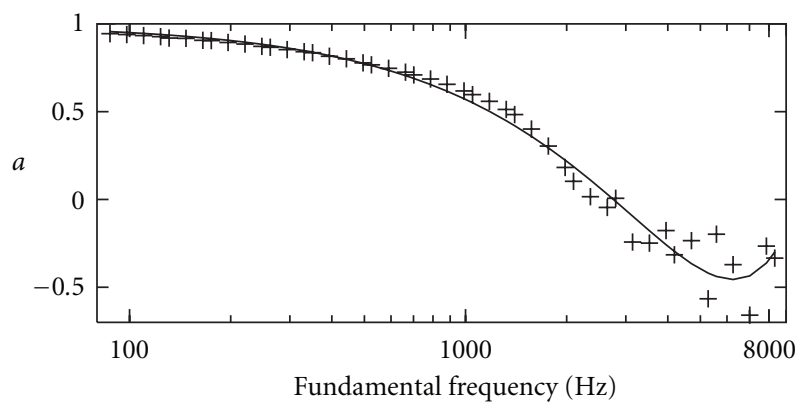

(e)

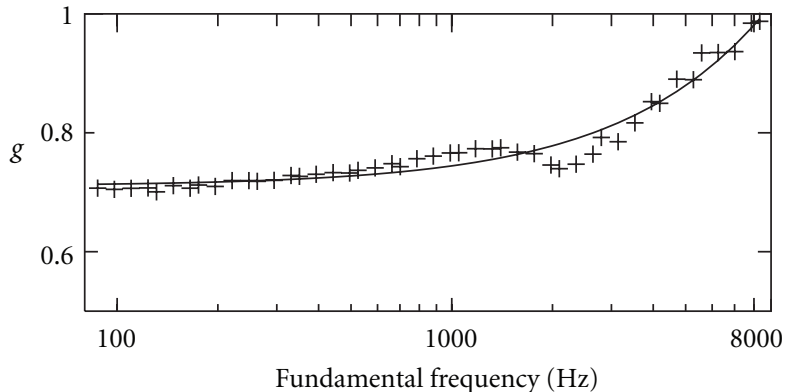

(b)

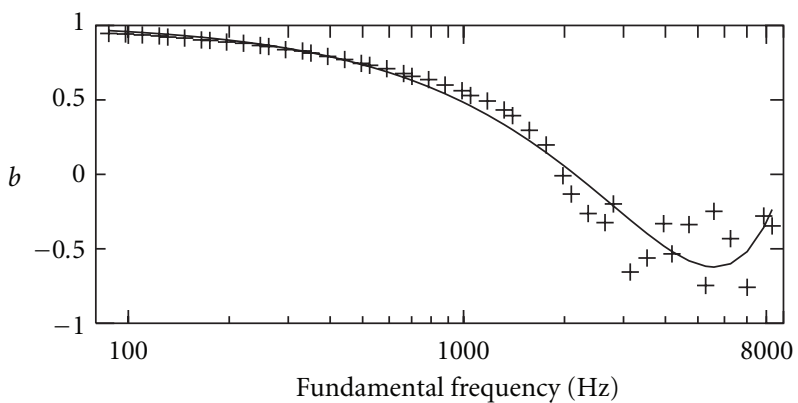

(d)

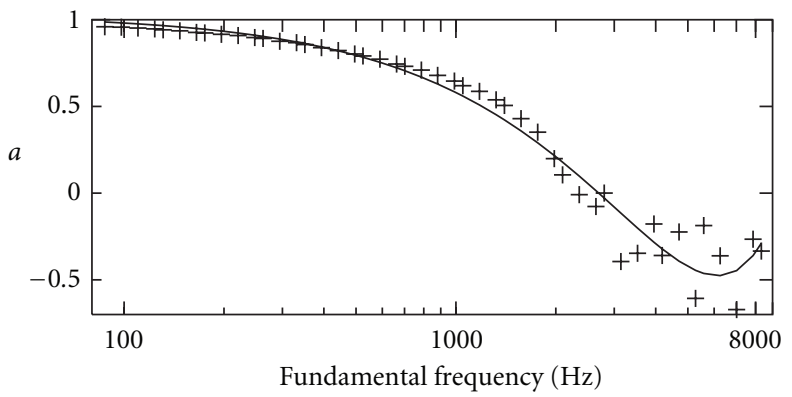

(f)

FIGURE 9: The gain factor $g$, the filter zero $b$, and the filter pole $a$ estimates (plus signs) for (a), (c), and (e) the third-order B-spline BLIT and (b), (d), and (f) the fourth-order B-spline BLEP, respectively, and the polynomial approximations of the filter parameters (solid lines).

TABLE 3: Coefficients of the polynomial fits for the first-order postequalising filter parameters for the ideally bandlimited sawtooth oscillator.

\begin{tabular}{lccc}
\hline$c_{i}$ & \multicolumn{3}{c}{ Filter parameter } \\
$i$ & $\hat{g}$ & $\hat{b}_{1}$ & $\hat{a}_{1}$ \\
\hline 0 & 0.5400 & 0.3894 & 0.6398 \\
1 & $4.473 \times 10^{-5}$ & $-3.102 \times 10^{-4}$ & $-2.417 \times 10^{-4}$ \\
2 & 0 & $2.417 \times 10^{-8}$ & $1.335 \times 10^{-8}$ \\
\hline
\end{tabular}

3.3. Filter Design Evaluation. Figure 11 shows the match of the waveforms of the filtered antialiasing sawtooth oscillators to the recorded Moog sawtooth oscillator output with fundamental frequencies of $220.62 \mathrm{~Hz}$ and $2.096 \mathrm{kHz}$. In Figures $11(\mathrm{a})$ and 11(b) are shown the filtered ideally bandlimited waveforms. Figures 11(c) and 11(d) depict the waveforms of the output of the post-equalising filter applied to the third-order B-spline BLIT oscillators. The filtered fourthorder B-spline BLEP waveforms are plotted in Figures 11(e) and $11(\mathrm{f})$. Figures $11(\mathrm{~g})$ and $11(\mathrm{~h})$ show the filtered secondorder DPW oscillator, and the filtered fourth-order DPW waveforms are plotted in Figures 11(i) and 11(j).

In Figure 11, it can be seen that the match of the filtered oscillator outputs depends on both the oscillator and the fundamental frequency. Therefore, as with the PD model, the root mean squared error and the maximum absolute harmonic magnitude error of the filtered oscillator outputs with respect to the respective recorded Moog sawtooth oscillator signals were computed using the polynomial approximations of the filter parameters, and the error measures are shown in Figure 12. Figures 12(a) and 12(b) depict the error measures for the filtered ideally bandlimited sawtooth with a solid line. In Figures 12(c) and 12(d), the RMSE and the maximum absolute harmonic magnitude error of the filtered thirdorder BLIT oscillator are plotted, respectively, with a solid line. In addition, the error measures of the filtered fourthorder BLEP sawtooth are plotted in Figures 12(c) and 12(d) with a dashed line. The error measures of the filtered second- 


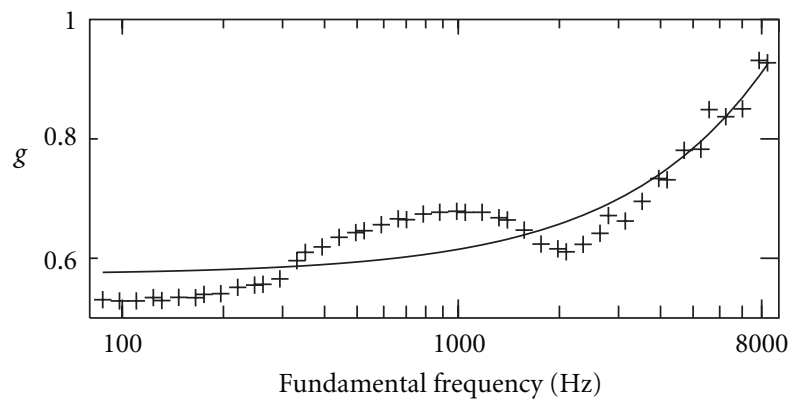

(a)

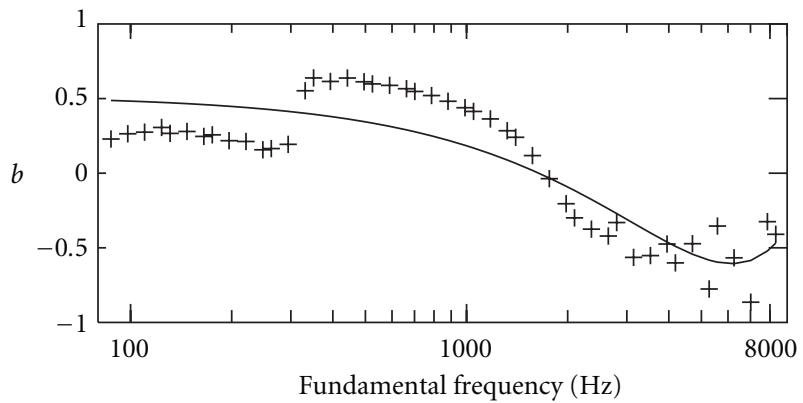

(c)

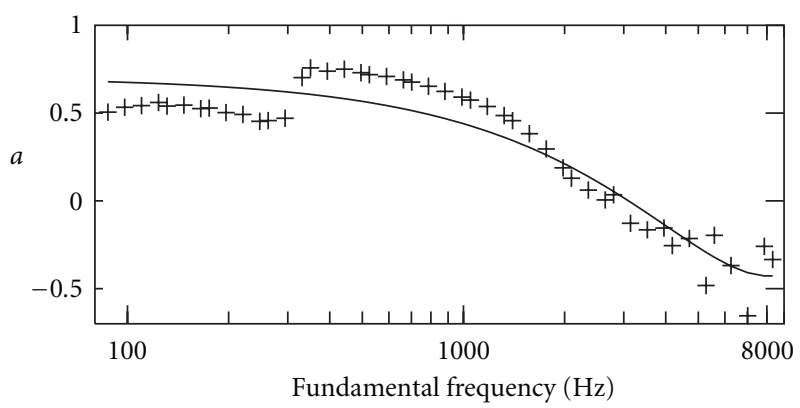

(e)

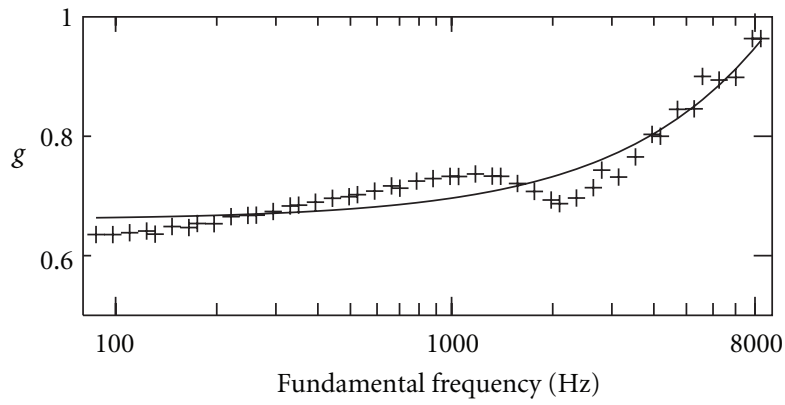

(b)

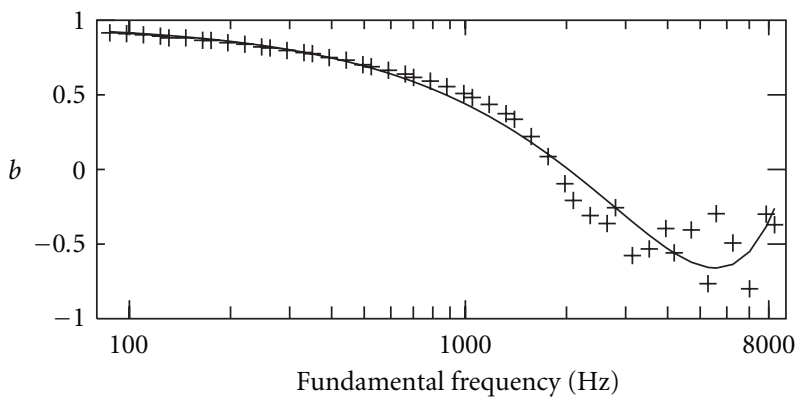

(d)

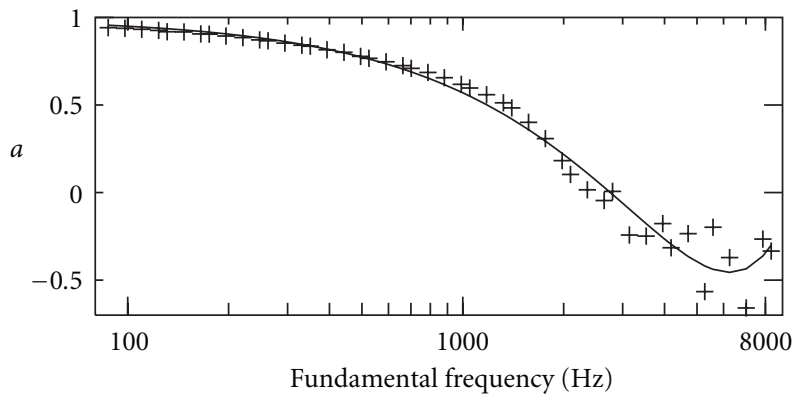

(f)

FIgURE 10: Filter parameter estimates (plus signs) of (a), (b) the gain factor $g$, (c), (d) the filter zero $b$, and (e), (f) the filter pole a for the second- and fourth-order DPW oscillator, respectively. The polynomial approximations of the filter parameters are given with solid lines.

and fourth-order DPW algorithms are shown in Figures $12(\mathrm{e})$ and $12(\mathrm{f})$ with a solid and a dashed line, respectively.

In Figure 12 it can be seen that the ideally bandlimited oscillator has the largest root mean squared error (RMSE) compared to the other oscillators (see Figures 12(a), 12(c), and $12(\mathrm{e})$ ). Moreover, the same observation can be made for the maximum absolute harmonic magnitude error (see Figures 12(b), 12(d), and 12(f)). The differences in the error measures are explained by the fact that all algorithms, other than the ideally bandlimited sawtooth oscillator, have inherently a spectral envelope that differs from the textbook sawtooth's $-6 \mathrm{~dB}$ per octave envelope $[15,16,32]$. The spectral envelope of the other antialiasing oscillator algorithms roll off faster than that of the textbook sawtooth, which means that their spectra are already closer to the spectrum of the Moog sawtooth oscillator prior to the filtering step (see Figures 1 and 2).

Again, as with the PD model, the polynomial approximations of the filter parameters yield errors that are comparable to the error obtained with the estimated parameter values, as indicated in Figure 13 for the filtered fourth-order BLEP oscillator. Similar observations were made for all tested antialiasing oscillator algorithms. As can be seen in Figures 13(a), the polynomial approximation of the filter parameters (solid line) has an RMSE that is at its maximum as bad as that of the tabulated parameter estimates (crosses). At very low fundamental frequencies, the polynomial approximation results in a larger maximum absolute harmonic magnitude error (see Figures 13(b)) than the estimated parameters, but at higher fundamental frequencies the difference between these approaches is small. Therefore, accuracy-wise the polynomial approximation provides as a good match as the tabulated parameter estimates. Again, the use of a fixed filter, that is, a filter whose parameters are independent from any synthesis control data like $f_{0}$, does not provide as good match as the $f_{0}$-dependent filters, as indicated in Figure 13 with circles using the filter parameters estimated for $f_{0}=524 \mathrm{~Hz}$.

Of the tested oscillator algorithms, an excellent performance is obtained with the third-order B-spline BLIT, the fourth-order B-spline BLEP, and the fourth-order DPW 


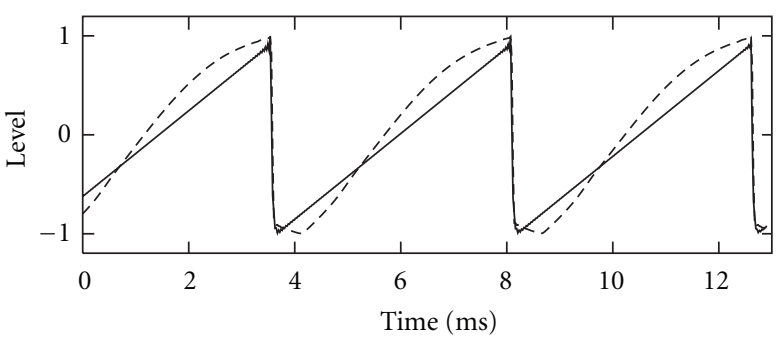

(a)

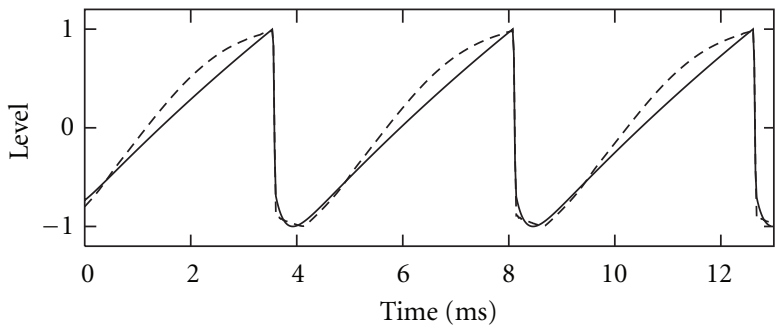

(c)

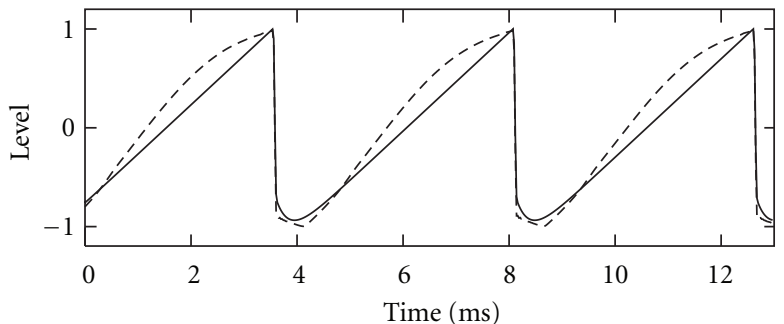

(e)

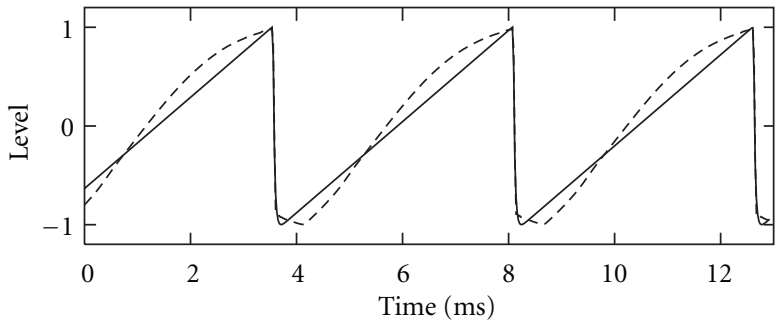

(g)

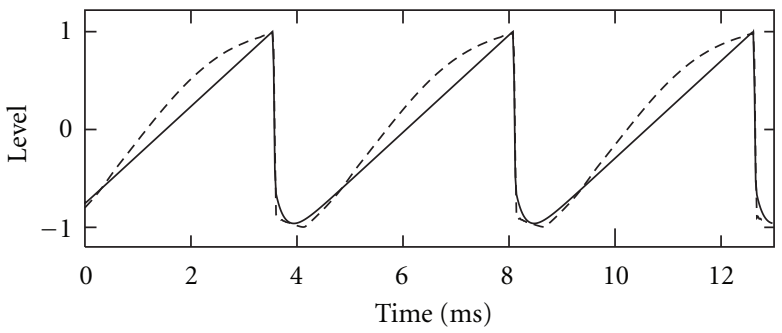

(i)

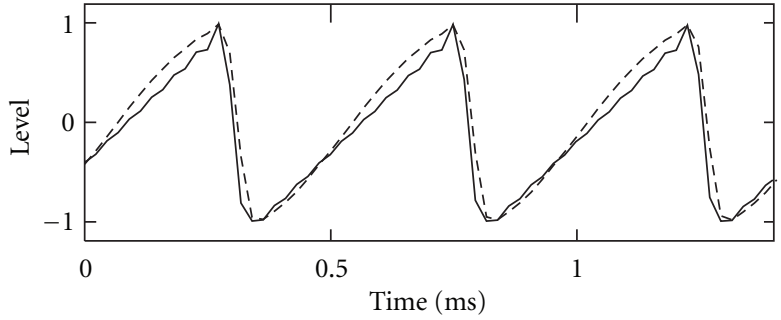

(b)

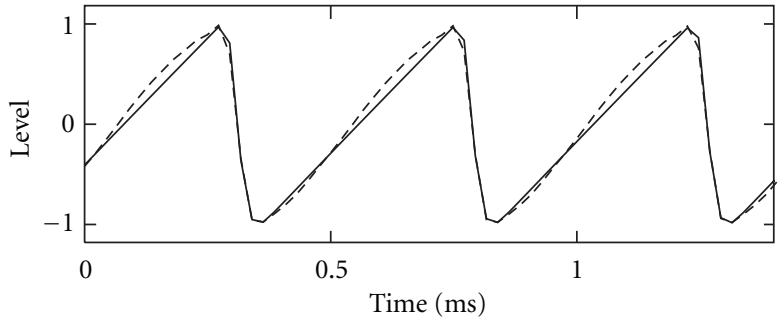

(d)

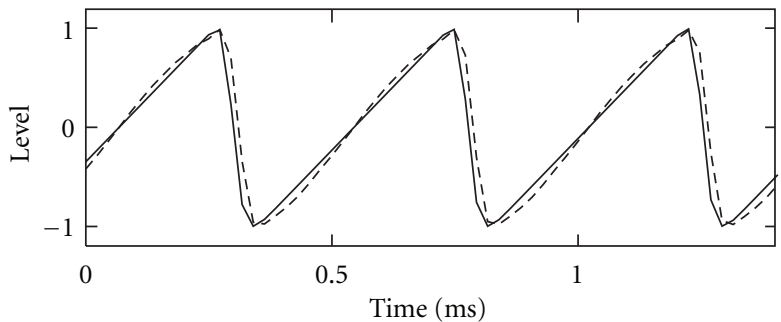

(f)

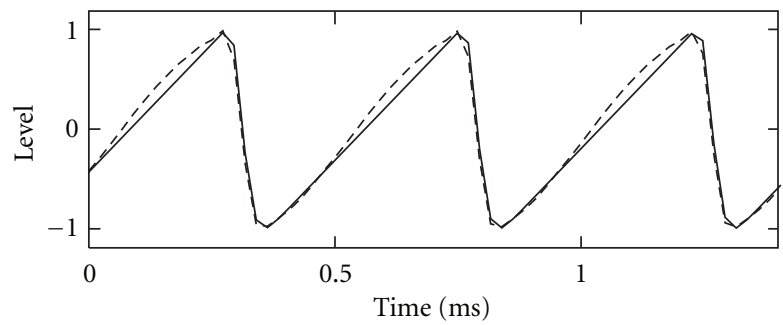

(h)

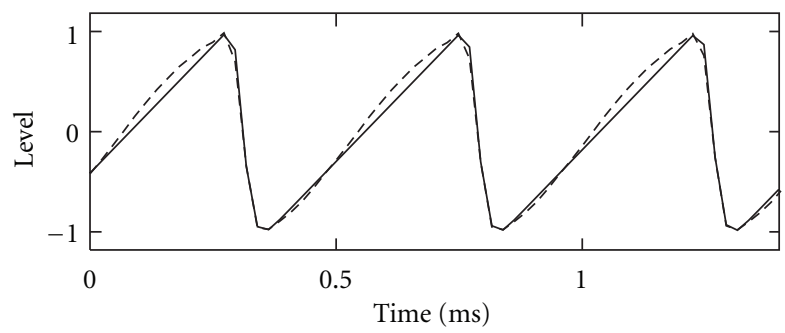

(j)

Figure 11: Waveforms of the filtered (a), (b) ideally bandlimited, (c), (d) third-order B-spline BLIT, (e), (f) third-order B-spline BLEP, (g), (h) second-order DPW, and (i), (j) fourth-order DPW sawtooth oscillator output at fundamental frequencies of $220.62 \mathrm{~Hz}$ and $2.096 \mathrm{kHz}$, respectively. The corresponding waveforms of the recorded signals are drawn with dashed line in all plots.

oscillators. Although these approaches have worse RMSE than the phase distortion model (dash-dotted line in Figures 12(c) and 12(e)), their maximum absolute harmonic magnitude errors are smaller than those of the phase distortion model (dash-dotted line in Figures 12(d) and 12(f)). In fact, their maximum absolute harmonic magnitude error is below $3 \mathrm{~dB}$ at all tested fundamental frequencies.

In Figure 12, it can be seen that the post-equalising filtering approach does match well to the analogue oscillator spectrum. Therefore, it can be concluded that the post-equalising 


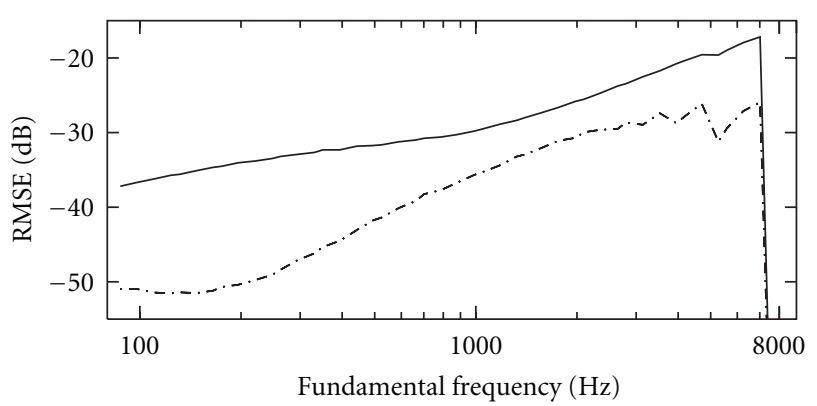

(a)

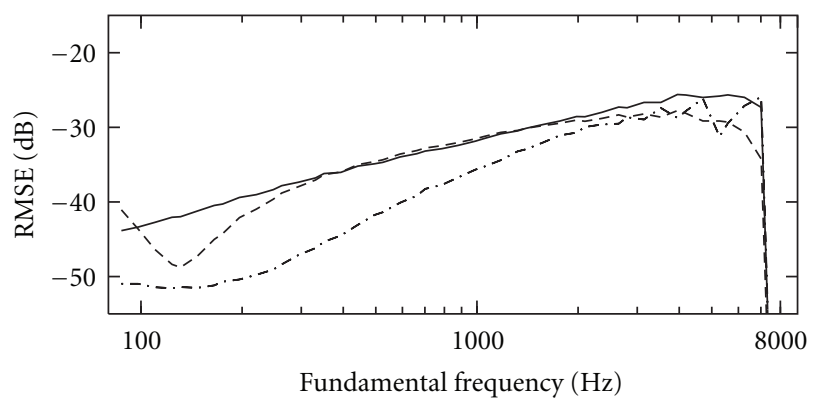

(c)

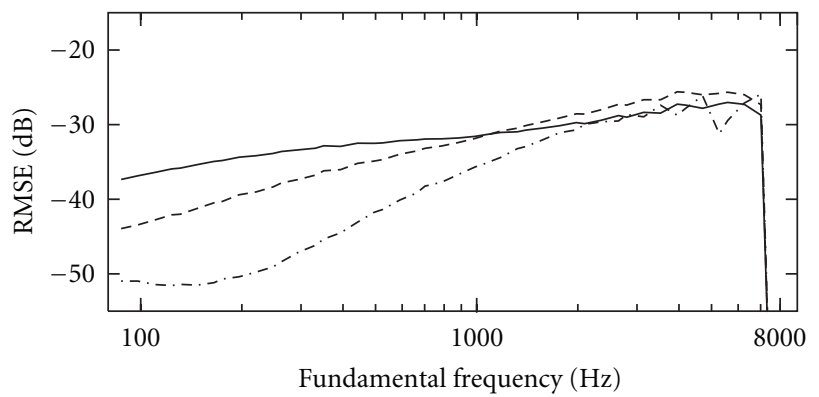

(e)

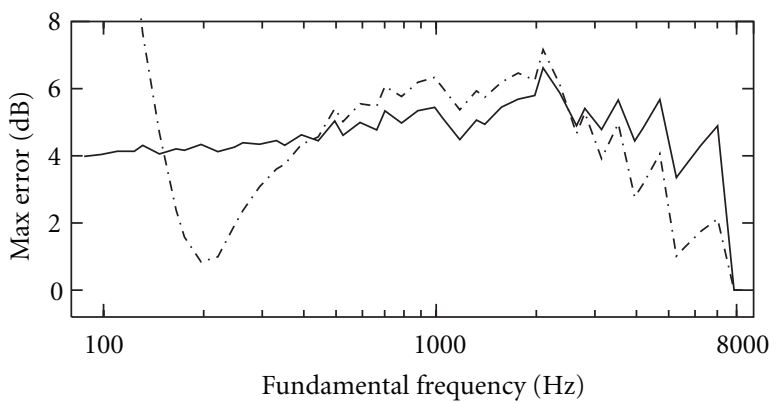

(b)

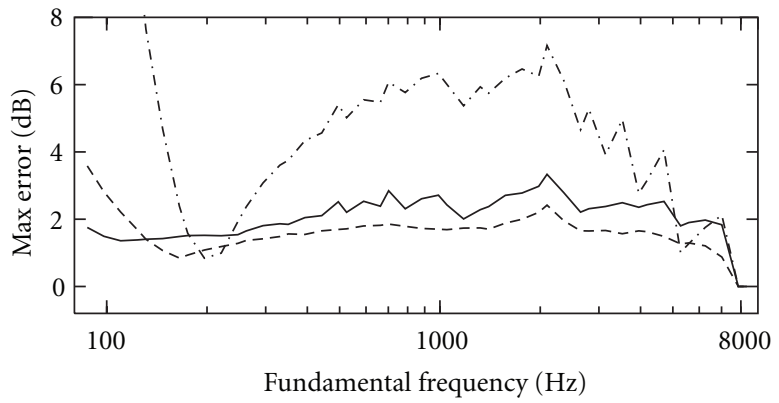

(d)

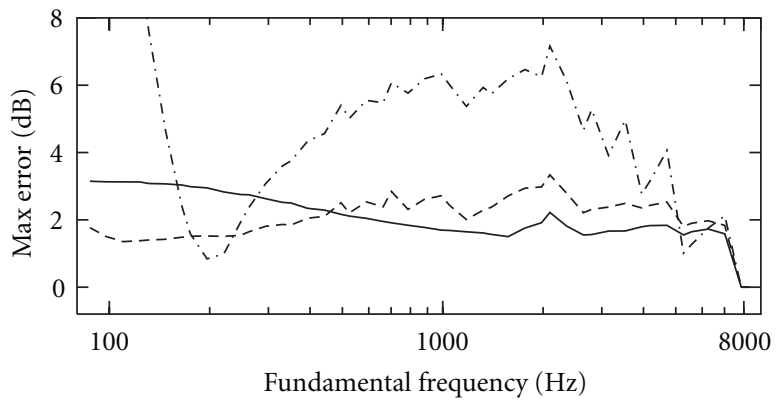

(f)

FIGURE 12: Root mean squared error (RMSE) and the maximum absolute harmonic magnitude error of the filtered (a), (b) the ideally bandlimited oscillator (solid line), (c), (d) the third-order B-spline BLIT (solid line) and the fourth-order B-spline BLEP (dashed line) oscillator, and (e), (f) the second- (solid line) and fourth-order (dashed line) DPW oscillator outputs, respectively. The dash-dotted line in all plots represents the corresponding error of the phase distortion model.

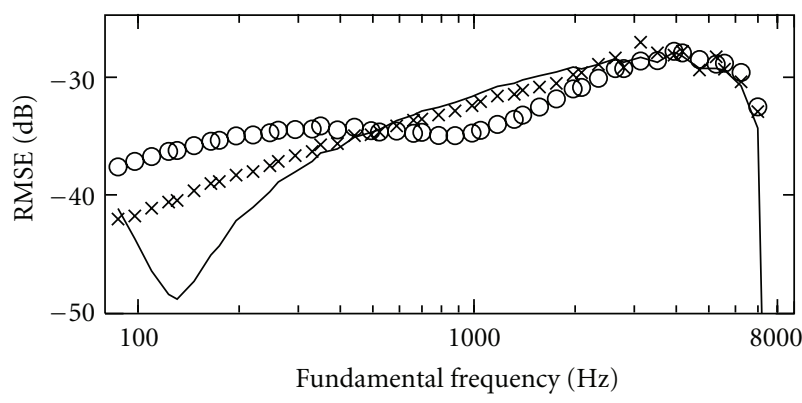

(a)

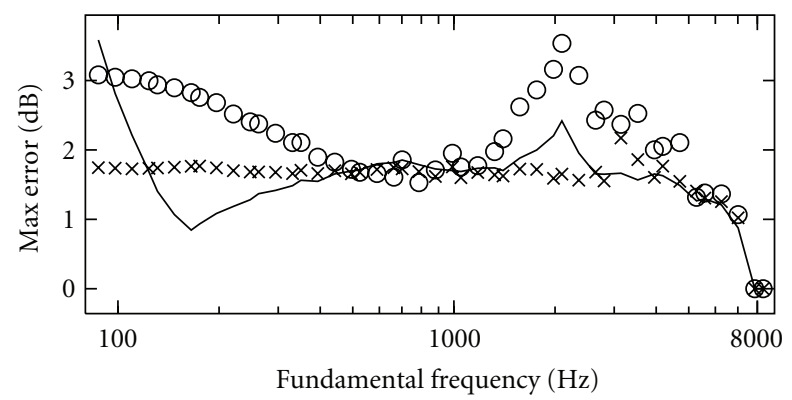

(b)

FIGURE 13: (a) RMSE and (b) the maximum absolute harmonic magnitude error of the filtered fourth-order B-spline BLEP sawtooth oscillator using the polynomial approximation of the filter parameters (solid line), the estimated parameters (crosses), and a fixed filter that has the parameters estimated for the fundamental frequency of $524 \mathrm{~Hz}$ (circles). 
filter approach is a valid model for the Moog sawtooth oscillator and that the proposed low-order polynomials can be used to compute the $f_{0}$-dependent filter parameters for the discussed algorithms. The best performance is obtained with the fourth-order B-spline BLEP sawtooth oscillator that has the smallest error measures in all tested fundamental frequencies. Moreover, since the fourth-order B-spline BLEP sawtooth oscillator has been demonstrated to be aliasing-free up to almost $8 \mathrm{kHz}$ [32], it can be considered to provide an excellent model for the Moog sawtooth oscillator. However, it should be noted that the $f_{0}$-dependent filter parameters result in a time-varying recursive filter that requires some attention in its realisation.

\section{Conclusions}

Previously, discrete-time modelling of the source waveforms used in subtractive sound synthesis has focused on reducing aliasing occurring in the waveform synthesis. The target of these oscillators has been the geometric textbook waveform, and attempts to model the actual waveform generated by an analogue oscillator have been neglected until recently. So far, only two papers have dealt with discrete-time modelling of an analogue oscillator. In this paper, two new discrete-time models for the Moog sawtooth oscillator suitable for realtime implementation were investigated.

The first approach was based on time-domain modelling of the recorded analogue waveforms using phase distortion synthesis. A simplified phase distortion function with two linear segments was used, and parameter estimation for the model was carried out. The parameter was noted to be dependent on the fundamental frequency, and a linear fit for the parameter estimates was computed. The model parameter fit was tested, and it was found to produce a faithful imitation of the recorded signals on a wide range of fundamental frequencies with errors that occur mainly at harmonics with very high frequency.

The second approach was based on frequency-domain matching of an antialiasing oscillator spectrum to the recorded analogue waveform spectrum using a postequalising filter. Five antialiasing oscillators were discussed as the source material, and a first-order IIR filter was considered as the equalising filter. For each oscillator algorithm, filter parameters were estimated, and low-order polynomial approximations of the parameters as a function of the fundamental frequency were fitted. The polynomial approximations were tested and the spectral errors between the filter outputs and the recorded signal were computed. It was found that the post-equalising filter provides an accurate match between the antialiasing oscillator and the recorded spectra, and that with a properly chosen source oscillator (the fourth-order B-spline BLEP oscillator), the spectral error can be reduced to be almost negligible.

Considering all the models discussed in this paper, the phase distortion model and the fourth-order B-spline BLEP oscillator filtered with a first-order IIR post-equalising filter provide the best match to the recorded analogue waveform. Even though they do have a mismatch to the recorded
TABLE 4: Coefficients of the polynomial fits for the first-order postequalising filter parameters for (a) the third-order B-spline BLIT, (b) the fourth-order BLEP, (c) the second-order DPW, and (d) the fourth-order DPW sawtooth oscillator.

(a)

\begin{tabular}{lccc}
\hline$c_{i}$ & \multicolumn{3}{c}{ Filter parameter } \\
$i$ & $\hat{g}$ & $\hat{b}_{1}$ & $\hat{a}_{1}$ \\
\hline 0 & 0.6599 & 0.9741 & 0.9963 \\
1 & $3.608 \times 10^{-5}$ & $-5.876 \times 10^{-4}$ & $-4.634 \times 10^{-4}$ \\
2 & 0 & $5.279 \times 10^{-8}$ & $3.696 \times 10^{-8}$ \\
\hline
\end{tabular}

(b)

\begin{tabular}{lccc}
\hline$c_{i}$ & \multicolumn{3}{c}{ Filter parameter } \\
$i$ & $\hat{g}$ & $\hat{b}_{1}$ & $\hat{a}_{1}$ \\
\hline 0 & 0.7105 & 1.0161 & 1.0294 \\
1 & $3.380 \times 10^{-5}$ & $-5.850 \times 10^{-5}$ & $-4.8921 \times 10^{-5}$ \\
2 & 0 & $5.220 \times 10^{-8}$ & $3.974 \times 10^{-8}$ \\
\hline
\end{tabular}

(c)

\begin{tabular}{lccc}
\hline$c_{i}$ & \multicolumn{3}{c}{ Filter parameter } \\
$i$ & $\hat{g}$ & $\hat{b}_{1}$ & $\hat{a}_{1}$ \\
\hline 0 & 0.5727 & 0.5192 & 0.7027 \\
1 & $4.230 \times 10^{-5}$ & $-3.650 \times 10^{-4}$ & $-2.806 \times 10^{-4}$ \\
2 & 0 & $2.959 \times 10^{-8}$ & $1.741 \times 10^{-8}$ \\
\hline
\end{tabular}

(d)

\begin{tabular}{lccc}
\hline$c_{i}$ & \multicolumn{3}{c}{ Filter parameter } \\
$i$ & $\hat{g}$ & $\hat{b}_{1}$ & $\hat{a}_{1}$ \\
\hline 0 & 0.6603 & 0.9736 & 0.9959 \\
1 & $3.600 \times 10^{-5}$ & $-5.871 \times 10^{-4}$ & $-4.630 \times 10^{-4}$ \\
2 & 0 & $5.272 \times 10^{-8}$ & $3.691 \times 10^{-8}$ \\
\hline
\end{tabular}

reference signals at some fundamental frequencies, they do provide almost bandlimited models of the Moog sawtooth waveform. Aliasing is suppressed better using the BLEP method than using the phase distortion model.

This work has demonstrated that the set of algorithms for virtual analogue oscillators can be expanded further to include specific oscillator types alongside the more generic models. Sound examples of the reference oscillator and the discussed techniques can be found online at http:// www . acoustics.hut.fi/go/jasp-moog/.

\section{Acknowledgments}

This work has been partly funded by the European Union as part of the 7th Framework Programme (SAME Project, ref. 215749) and by the Academy of Finland (Project no. 122815).

\section{References}

[1] H. F. Olson and H. Belar, "Electronic music synthesis," Journal of the Acoustical Society of America, vol. 27, no. 3, pp. 595-612, 1955. 
[2] J. Lane, D. Hoory, E. Martinez, and P. Wang, "Modeling analog synthesis with DSPs," Computer Music Journal, vol. 21, no. 4, pp. 23-41, 1997.

[3] R. A. Moog, "A voltage-controlled low-pass high-pass filter for audio signal processing," in Proceedings of the 17th Audio Engineering Society Convention, New York, NY, USA, October 1965 , preprint 413.

[4] T. Stilson and J. O. Smith, "Analyzing the Moog VCF with considerations for digital implementation," in Proceedings of the International Computer Music Conference, pp. 398-401, Hong Kong, China, August 1996.

[5] F. Fontana, "Preserving the structure of the Moog VCF in the digital domain," in Proceedings of the International Computer Music Conference, pp. 291-294, Thessaloniki, Greece, September 1997.

[6] A. Huovilainen, "Non-linear digital implementation of the Moog ladder filter," in Proceedings of the 7th International Conference on Digital Audio Effects (DAFx '04), pp. 61-64, Naples, Italy, September 2004.

[7] V. Välimäki and A. Huovilainen, "Oscillator and filter algorithms for virtual analog synthesis," Computer Music Journal, vol. 30, no. 2, pp. 19-31, 2006.

[8] T. E. Stinchcombe, "Analysis of the Moog transistor ladder and derivative filters," 2008, http://www.timstinchcombe.co.uk/ synth/Moog_ladder_tf.pdf.

[9] T. Hélie, "On the use of Volterra series for real-time simulations of weakly nonlinear analog audio devices: application to the Moog ladder filter," in Proceedings of the 9th International Conference on Digital Audio Effects (DAFx '06), pp. 7-12, Montréal, Canada, September 2006.

[10] T. Hélie, "Volterra series and state transformation for realtime simulations of audio circuits including saturations: application to the Moog ladder filter," IEEE Transactions on Audio, Speech and Language Processing, vol. 18, no. 4, pp. 747759, 2010.

[11] M. Civolani and F. Fontana, "A nonlinear digital model of the EMS VCS3 voltage-controlled filter," in Proceedings of the 11th International Conference on Digital Audio Effects (DAFx '08), pp. 35-42, Espoo, Finland, September 2008.

[12] F. Fontana and M. Civolani, "Modeling of the EMS VCS3 voltage-controlled filter as a nonlinear filter network," IEEE Transactions on Audio, Speech and Language Processing, vol. 18, no. 4, pp. 760-772, 2010.

[13] A. Huovilainen, Design of a scalable polyphony-MIDI synthesizer, M.S. thesis, Aalto University School of Science and Technology, Espoo, Finland, May 2010, http://lib.tkk.fi/ Dipl/2010/urn100219.pdf.

[14] V. Välimäki and A. Huovilainen, "Antialiasing oscillators in subtractive synthesis," IEEE Signal Processing Magazine, vol. 24, no. 2, pp. 116-125, 2007.

[15] J. Nam, V. Välimäki, J. S. Abel, and J. O. Smith, "Efficient antialiasing oscillator algorithms using low-order fractional delay filters," IEEE Transactions on Audio, Speech, and Language Processing, vol. 18, no. 4, pp. 773-785, 2010.

[16] V. Välimäki, J. Nam, J. O. Smith, and J. S. Abel, "Aliassuppressed oscillators based on differentiated polynomial waveforms," IEEE Transactions on Audio, Speech and Language Processing, vol. 18, no. 4, pp. 786-798, 2010.

[17] H. Chamberlin, Musical Applications of Microprocessors, chapter 13, World Scientific, Hackensack, NJ, USA, 2nd edition, 1985.

[18] J. Kleimola, Design and implementation of a software sound synthesizer, M.S. thesis, TKK Helsinki University of Tech- nology, Espoo, Finland, 2005, http://www.acoustics.hut.fi/ publications/files/theses/kleimola_mst/.

[19] J. Kleimola, V. Lazzarini, J. Timoney, and V. Välimäki, "Phaseshaping oscillator algorithms for musical sound synthesis," in Proceedings of the 7th Sound and Music Computing Conference, pp. 94-101, Barcelona, Spain, July 2010.

[20] Moog Music Inc., "Minimoog Voyager Old School User's Guide,” Asheville, NC, USA, 2008, http://www.bigbriar.com/ manuals/old_school_manual_1_0.pdf.

[21] R. A. Moog, "Voltage-controlled electronic music modules," in Proceedings of the 16th Audio Engineering Society Convention, New York, NY, USA, October 1964, preprint 346. Reprinted in Journal of Audio Engineering Society, vol. 13, no. 3, pp. 200206, 1965.

[22] G. De Sanctis and A. Sarti, "Virtual analog modeling in the wave-digital domain," IEEE Transactions on Audio, Speech and Language Processing, vol. 18, no. 4, pp. 715-727, 2010.

[23] M. Ishibashi, "Electronic music instrument," U.S. patent no. 4,658,691, 1987.

[24] J. Timoney, V. Lazzarini, J. Pekonen, and V. Välimäki, "Spectrally rich phase distortion sound synthesis using an allpass filter," in Proceedings of the IEEE International Conference on Acoustics, Speech, and Signal Processing (ICASSP '09), pp. 293296, Taipei, Taiwan, April 2009.

[25] V. Lazzarini, J. Timoney, J. Pekonen, and V. Välimäki, "Adaptive phase distortion synthesis," in Proceedings of the 12th International Conference on Digital Audio Effects (DAFx '09), pp. 28-35, Como, Italy, September 2009.

[26] V. Lazzarini and J. Timoney, "New perspectives on distortion synthesis for virtual analog oscillators," Computer Music Journal, vol. 34, no. 1, pp. 28-40, 2010.

[27] E. Zwicker and H. Fastl, Psychoacoustics, Springer, Berlin, Germany, 1990.

[28] A. Chaudhary, "Bandlimited simulation of analog synthesizer modules by additive synthesis," in Proceedings of the 105th Audio Engineering Society Convention, San Francisco, Calif, USA, October 1998, preprint 4779.

[29] P. Burk, "Band limited oscillators using wave table synthesis," in Audio Anecdotes II-Tools, Tips, and Techniques for Digital Audio, K. Greenebaum and R. Barzel, Eds., pp. 37-53, A. K. Peters, Ltd, Wellesley, Mass, USA, 2004.

[30] T. Stilson and J. O. Smith, "Alias-free digital synthesis of classic analog waveforms," in Proceedings of the International Computer Music Conference, pp. 332-335, Hong Kong, China, August 1996.

[31] E. Brandt, "Hard sync without aliasing," in Proceedings of the International Computer Music Conference, pp. 365-368, Havana, Cuba, September 2001.

[32] V. Välimäki, J. Pekonen, and J. Nam, "Perceptually informed synthesis of bandlimited classical waveforms using integrated polynomial interpolation," Journal of the Acoustical Society of America, in press.

[33] A. B. Leary and C. T. Bright, "Bandlimited digital synthesis of analog waveforms," U.S. patent no. 7,589,272, September 2009.

[34] V. Välimäki, "Discrete-time synthesis of the sawtooth waveform with reduced aliasing," IEEE Signal Processing Letters, vol. 12, no. 3, pp. 214-217, 2005.

[35] S. K. Mitra, Digital Signal Processing: A Computer Based Approach, McGraw-Hill, New York, NY, USA, 3rd edition, 2005. 